\title{
Disponibilidad de Leche de los Países Sudamericanos en las Últimas Cinco Décadas: Elementos para Análisis y Perspectivas Futuras
}

\author{
Luis F. Restrepo-Betancur ${ }^{(1)}$, Carolina Peña-Serna ${ }^{(2) *}$, y Natalia Zapata-López ${ }^{(3)}$ \\ (1) Grupo de Innovaciones Socioeconómicas Rurales (GISER). Facultad de Ciencias Agrarias, Universidad de \\ Antioquia, Medellín, Colombia. (e-mail: frbstatistical@yahoo.es) \\ (2) Grupo de Investigación en Ciencias Agrarias (GRICA). Facultad de Ciencias Agrarias, Universidad de Antioquia, \\ Medellín, Colombia. (e-mail: carolina.pena@udea.edu.co) \\ (3) Grupo de Investigación en Alimentación y Nutrición Humana (GIANH). Escuela de Nutrición y Dietética, Universidad \\ de Antioquia, Medellín, Colombia. (e-mail: nyaneth.zapata@udea.edu.co) \\ * Autor a quien debe ser dirigida la correspondencia
}

Recibido Sep. 14, 2018; Aceptado Dic. 5, 2018; Versión final Feb. 24, 2019, Publicado Ago. 2019

\begin{abstract}
Resumen
El objetivo del estudio fue comparar la cantidad per cápita de leche disponible entre países de Sudamérica durante las últimas cinco décadas. Se analizaron los datos reportados en las hojas de balance de alimentos de la FAO a través del modelo lineal general con contraste de Tukey e igualmente se utilizó estadística descriptiva exploratoria de tipo unidimensional y análisis de Cluster y Biplot. Se presentaron diferencias entre países de Sudamérica en lo relacionado con la cantidad de leche disponible para consumo humano. Uruguay y Argentina mostraron mayor disponibilidad a lo largo del periodo mientras que Bolivia, Perú y Paraguay menor. Se encontró una brecha entre la cantidad de leche disponible y la recomendación nutricional de consumo del alimento. Ninguno de los países de la región alcanzó a cubrir las recomendaciones nutricionales internacionales, a pesar de que la mayoría de ellos incrementó la cantidad de leche disponible a lo largo del tiempo.
\end{abstract}

Palabras clave: disponibilidad de leche; producción de leche; consumo de leche; salud humana; nutrición

\section{Milk Supply of the South American Countries in the Last Five Decades: Basis for Analysis and Future Perspectives}

\begin{abstract}
The aim of this study was to compare the per capita milk supply between South American countries during the last five decades. The data in the food balance sheets reported by the FAO were analyzed through a general lineal model with Tukey contrast as well as one-dimensional exploratory descriptive statistics, Cluster and Biplot analyses. The South American countries exhibited differences on the quantity of milk supply for human consumption. Uruguay and Argentina showed the greater milk supply throughout the period while Bolivia, Peru and Paraguay the lower. There is a gap between the quantity of milk supply and the nutritional needs of the foodstuff. Despite the majority of the South American countries increased the quantity of milk along time, none of them achieved the international nutritional recommendation.
\end{abstract}




\section{INTRODUCCIÓN}

Se entiende como leche la secreción líquida de las glándulas mamarias de los mamíferos hembras. Tradicionalmente, los humanos ingieren leche materna en etapas iniciales de la vida, y leche de vaca, de búfala, de cabra, entre otras especies, en etapas posteriores (Pereira, 2014). No obstante, la leche de vaca es la de mayor consumo alrededor del mundo con una ingesta total del $85 \%$, seguida por la leche de búfala con $11 \%$, cabra con $2 \%$ y otras especies $2 \%$ (Secretaría de Economía-Dirección General de Industrias Básicas, 2012). En los humanos, después del primer año de vida, la leche de vaca es un importante alimento para la salud ya que contiene una relación adecuada de energía y nutrientes que se constituyen en elementos claves para el crecimiento y desarrollo óseo, manejo y control del peso, entre otros beneficios (Rozenberg, 2016; Lu et al., 2018). Estudios epidemiológicos demuestran que la composición nutricional de la leche de vaca con su proteína de alto valor biológico y fuente importante de calcio tienen un efecto protector en la salud cardiovascular y metabólica (Abedini et al., 2015; Pereira y Vicente, 2017) adicional a mecanismos que protegen contra el cáncer (Méndez et al., 2017).

En los últimos años el sector lácteo a nivel mundial, incluyendo la producción de leche de búfala (Simanca et al., 2013), ha exhibido un crecimiento importante, no obstante, los países desarrollados y aquellos en vía de desarrollo presentan gran disparidad en términos de producción de leche (FAO y FEPALE, 2012). De acuerdo con la Organización de las Naciones Unidas para la Alimentación y la Agricultura (FAO) en 2016 la producción mundial de leche fue de 796,2 millones de toneladas de los cuales Asia contribuyó con 39,4\%, seguido por Europa 27,7\% y Norteamérica 13\%, siendo India, la Unión Europea y Estados Unidos las principales regiones productoras. Por el contrario, Centroamérica es la región con la menor producción, cercana a 2,2\% (FAO, 2018). Respecto a la disponibilidad de leche de vaca para consumo humano, la FAO (FAOSTAT, 2019) reporta que en el 2013 la población mundial tuvo una disponibilidad per cápita de 246,6g/persona-día, no obstante, Norteamérica, Europa y Oceanía presentaron una disponibilidad 2,5 veces mayor al promedio mundial. Hasta la fecha, no existe en la literatura estudios que muestren y comparen la disponibilidad de leche de vaca para consumo humano en los países de Sudamérica y que sirva como base para el análisis y la planificación de estrategias y políticas relacionadas con la producción, importación, exportación y canales de distribución que puedan garantizar el acceso y fomentar el consumo del alimento para beneficio de la población dada su importancia para la salud humana. Por esta razón, el presente estudio tuvo como objetivo analizar la disponibilidad de leche de vaca en los países de Sudamérica a fin de contrastar si hay diferencias significativas en la disponibilidad y así poder discutir las repercusiones sobre la salud humana, además de evidenciar las problemáticas actuales y contribuir a la toma de decisiones que permitan mejorar la producción de leche y fomentar su consumo en beneficio de la población.

\section{METODOLOGÍA}

Se realizó un estudio retrospectivo longitudinal con fuentes de información secundaria. Los datos analizados se recopilaron de las hojas de balance de alimentos reportadas por la FAO para los países de América del Sur en los años de 1960 a 2009. La hoja de balance de alimentos es una metodología que evalúa la disponibilidad de alimentos a nivel nacional y relaciona las entradas y salidas de estos que se encuentra potencialmente disponible para el consumo humano en un periodo de tiempo. La variable de estudio, disponibilidad per cápita de leche, fue de tipo cuantitativo continuo, expresada en g/persona-día y definida como la disponibilidad de leche total para consumo humano y aquella destinada a la elaboración de productos lácteos excepto para mantequilla.

La evaluación de la disponibilidad per cápita de leche se efectuó a través de un análisis descriptivo comparativo en las últimas cinco décadas (60,70, 80, 90 y primera del 2000), como variable control se tuvieron en cuenta los países de la región de América del Sur: Argentina (Arg), Bolivia (Bol), Brasil (Bra), Chile (Chi), Colombia (Col), Ecuador (Ecu), Paraguay (Par), Perú (Per), Uruguay (Uru) y Venezuela (Ven) y la variable de tipo cuantitativo continuo se asoció a la distribución probabilística normal mientras los países y las décadas evaluadas siguieron un patrón multinomial. El análisis estadístico de los datos se realizó mediante el modelo lineal general con contraste de Tukey al $5 \%$ de significancia, complementándose con análisis de Cluster y la técnica multivariada Biplot complementados con estadística descriptiva de tipo unidimensional. Dichos análisis fueron realizados usando los programas estadísticos SAS UNIVERSITY y R v.3.3.2 (de uso libre).

Para la discusión de resultados, los autores reconocen que, si bien algunos alimentos de origen vegetal y unos tipos de pescado tienen un contenido importante de calcio, es la leche y los productos derivados de esta, la fuente principal y más biodisponible del mineral. Por esta razón, en el presente estudio se analiza la disponibilidad de leche en el mismo sentido de disponibilidad de calcio, reconociendo que se dejan por fuera algunos alimentos que lo contienen, pero siendo consistentes con la literatura que afirma que estos aportan entre el $60-80 \%$ del nutriente en la dieta humana. 


\section{RESULTADOS}

Seis de los países analizados (Argentina, Bolivia, Brasil, Chile, Colombia y Paraguay) presentaron un aumento en la disponibilidad per cápita de leche entre la década del 60 y la primera del 2000, donde Brasil, Paraguay y Bolivia mostraron mayor incremento con valores que ascienden a $73,8 \%, 56,8 \%$ y $50,5 \%$ respectivamente. Por otro lado, Ecuador, Perú, Uruguay y Venezuela disminuyeron la disponibilidad per cápita con cifras en el orden de $11,8 \%, 20,3 \%, 11,5 \%$ y $10,3 \%$ respectivamente. Es de resaltar que a lo largo de las cinco décadas, Uruguay y Argentina fueron los países que exhibieron la mayor disponibilidad per cápita de leche con valores superiores a $400 \mathrm{~g} /$ persona-día mientras que Bolivia fue el país que presentó la menor disponibilidad por debajo de 94g/persona-día (Tabla 1).

Tabla 1: Análisis descriptivo comparativo de disponibilidad per cápita de leche por década en países de Sudamérica. Letras distintas en la columna indican diferencia estadística significativa $(p<0,05)$

\begin{tabular}{|c|c|c|c|c|c|}
\hline \multicolumn{5}{|c|}{ Disponibilidad de leche (g/persona-día) } \\
\hline \multirow{2}{*}{ País } & \multicolumn{5}{|c|}{ Décadas } \\
\cline { 2 - 6 } & 60 & 70 & 80 & 90 & 2000 \\
\hline Arg & $413,2 \pm 31 \mathrm{~b}$ & $469,2 \pm 19 \mathrm{a}$ & $468,5 \pm 30 \mathrm{a}$ & $567,7 \pm 62 \mathrm{a}$ & $518,7 \pm 69 \mathrm{a}$ \\
\hline Bol & $62,0 \pm 9 \mathrm{~g}$ & $83,1 \pm 13 \mathrm{e}$ & $91,8 \pm 16 \mathrm{e}$ & $92,3 \pm 16 \mathrm{f}$ & $93,3 \pm 15 \mathrm{~g}$ \\
\hline Bra & $188,2 \pm 12 \mathrm{e}$ & $196,2 \pm 20 \mathrm{~d}$ & $231,5 \pm 18 \mathrm{c}$ & $286,2 \pm 28 \mathrm{c}$ & $327 \pm 21 \mathrm{c}$ \\
\hline Chi & $225,4 \pm 14 \mathrm{~d}$ & $228,6 \pm 13 \mathrm{~d}$ & $232,1 \pm 14 \mathrm{c}$ & $304,1 \pm 28 \mathrm{c}$ & $284,6 \pm 17 \mathrm{~d}$ \\
\hline Col & $244,8 \pm 10 \mathrm{~d}$ & $203,9 \pm 27 \mathrm{~d}$ & $224,5 \pm 7 \mathrm{c}$ & $263,3 \pm 28 \mathrm{c}$ & $350,6 \pm 11 \mathrm{c}$ \\
\hline Ecu & $255,0 \pm 7 \mathrm{~d}$ & $259,8 \pm 5 \mathrm{c}$ & $190,7 \pm 12,7 \mathrm{c}$ & $249,0 \pm 28,2 \mathrm{~d}$ & $225,2 \pm 4 \mathrm{e}$ \\
\hline Par & $116,1 \pm 14 \mathrm{f}$ & $114,9 \pm 12 \mathrm{e}$ & $136,5 \pm 3 \mathrm{~d}$ & $205,6 \pm 39 \mathrm{e}$ & $182,1 \pm 8 f$ \\
\hline Per & $177,1 \pm 30 \mathrm{e}$ & $194,8 \pm 13 \mathrm{~d}$ & $162,4 \pm 15 \mathrm{~d}$ & $135,4 \pm 12 \mathrm{e}$ & $141,2 \pm 8 f$ \\
\hline Uru & $517,7 \pm 37 \mathrm{a}$ & $494,5 \pm 29 \mathrm{a}$ & $491,3 \pm 23 \mathrm{a}$ & $512,6 \pm 48 \mathrm{~b}$ & $458,3 \pm 76 \mathrm{~b}$ \\
\hline Ven & $289,2 \pm 25 \mathrm{c}$ & $340,6 \pm 66 \mathrm{~b}$ & $367,7 \pm 48 \mathrm{~b}$ & $252,5 \pm 21 \mathrm{e}$ & $259,2 \pm 73 \mathrm{e}$ \\
\hline
\end{tabular}

Argentina, Chile y Paraguay presentaron el mayor incremento en la disponibilidad de leche entre la década de los 80 a los 90; a diferencia de Colombia y Brasil que tuvieron el principal aumento entre la década de los 90 a la primera del 2000. De igual forma, países como Bolivia, Perú y Venezuela presentaron el mayor incremento en la disponibilidad de leche entre la década del 60 y 70 ; no obstante, Perú y Venezuela exhibieron una tendencia decreciente a partir de la década del 70 y 80 respectivamente (Tabla 2).

Tabla 2: Análisis comparativo de disponibilidad per cápita de leche por país para las décadas evaluadas. Letras distintas en la fila indican diferencia estadística significativa $(p<0,05)$

\begin{tabular}{|l|c|c|c|c|c|}
\hline \multirow{2}{*}{ País } & \multicolumn{5}{|c|}{ Década } \\
\cline { 2 - 6 } & 60 & 70 & 80 & 90 & 2000 \\
\hline Arg & $\mathrm{c}$ & $\mathrm{bc}$ & $\mathrm{bc}$ & $\mathrm{a}$ & $\mathrm{ab}$ \\
\hline Bol & $\mathrm{b}$ & $\mathrm{a}$ & $\mathrm{a}$ & $\mathrm{a}$ & $\mathrm{a}$ \\
\hline Bra & $\mathrm{d}$ & $\mathrm{d}$ & $\mathrm{c}$ & $\mathrm{b}$ & $\mathrm{a}$ \\
\hline Chi & $\mathrm{b}$ & $\mathrm{b}$ & $\mathrm{b}$ & $\mathrm{a}$ & $\mathrm{a}$ \\
\hline Col & $\mathrm{c}$ & $\mathrm{d}$ & $\mathrm{d}$ & $\mathrm{b}$ & $\mathrm{a}$ \\
\hline Ecu & $\mathrm{a}$ & $\mathrm{b}$ & $\mathrm{a}$ & $\mathrm{a}$ & $\mathrm{a}$ \\
\hline Par & $\mathrm{b}$ & $\mathrm{b}$ & $\mathrm{b}$ & $\mathrm{a}$ & $\mathrm{a}$ \\
\hline Per & $\mathrm{ab}$ & $\mathrm{a}$ & $\mathrm{bc}$ & $\mathrm{d}$ & $\mathrm{cd}$ \\
\hline Uru & $\mathrm{a}$ & $\mathrm{a}$ & $\mathrm{a}$ & $\mathrm{a}$ & $\mathrm{a}$ \\
\hline Ven & $\mathrm{bc}$ & $\mathrm{ab}$ & $\mathrm{a}$ & $\mathrm{c}$ & $\mathrm{c}$ \\
\hline
\end{tabular}

De acuerdo con la Tabla 2, la mayoría de países con excepción de Ecuador, Perú, Uruguay y Venezuela presentaron una tendencia creciente en la disponibilidad per cápita de leche a lo largo de las cinco décadas evaluadas. Sin embargo, Argentina mostró un descenso en la disponibilidad entre la década del 90 y la primera del 2000. En el análisis Biplot (Fig. 1) se puede observar que Argentina y Uruguay se alejan geométricamente 
de los demás países mostrando así que fueron los de mayor disponibilidad per cápita durante todas las décadas evaluadas. Por otro lado, Bolivia, Perú y Paraguay fueron las naciones que mostraron menor disponibilidad de leche. De igual forma en la Fig. 1 se puede apreciar una relación más estrecha entre las décadas del 60, 70 y 80 (D60, D70 y D80) en términos de cantidad de leche disponible para todos los países de Sudamérica mostrando un comportamiento creciente a partir de la década del 90 (D90 y D2000).

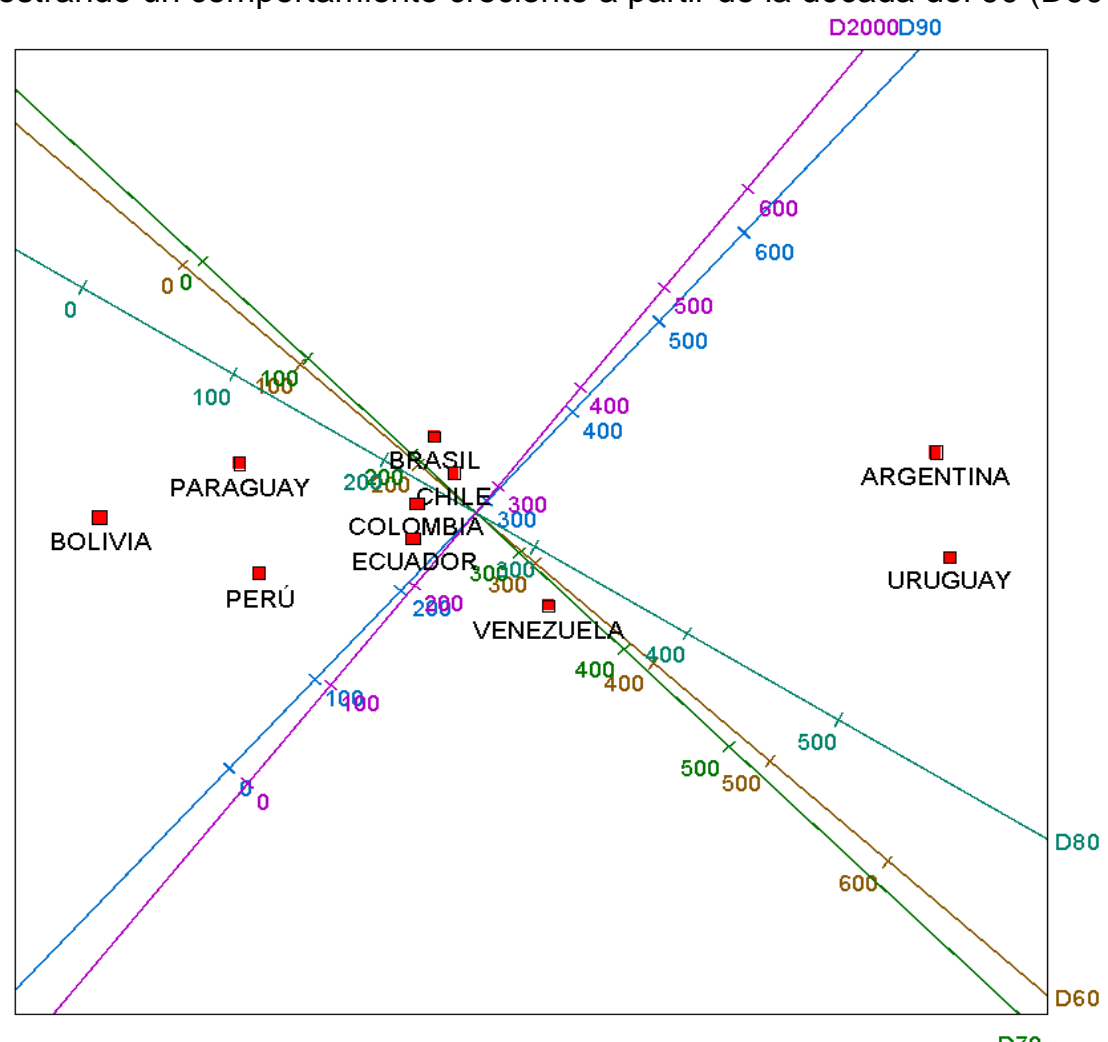

Fig. 1: Análisis Biplot de disponibilidad per cápita de leche en países de Sudamérica para décadas del 60 al 2000

Finalmente, cuando se evaluaron todas las décadas de forma simultánea, el procedimiento Cluster (Fig. 2) permitió definir cuatro grupos organizados según sus semejanzas en la disponibilidad de leche, agrupándolos así: grupo 1 conformado por Bolivia, grupo 2 integrado por Paraguay y Perú, grupo 3 constituido por Argentina y Uruguay, y, grupo 4 compuesto por Brasil, Chile, Colombia, Ecuador y Venezuela.

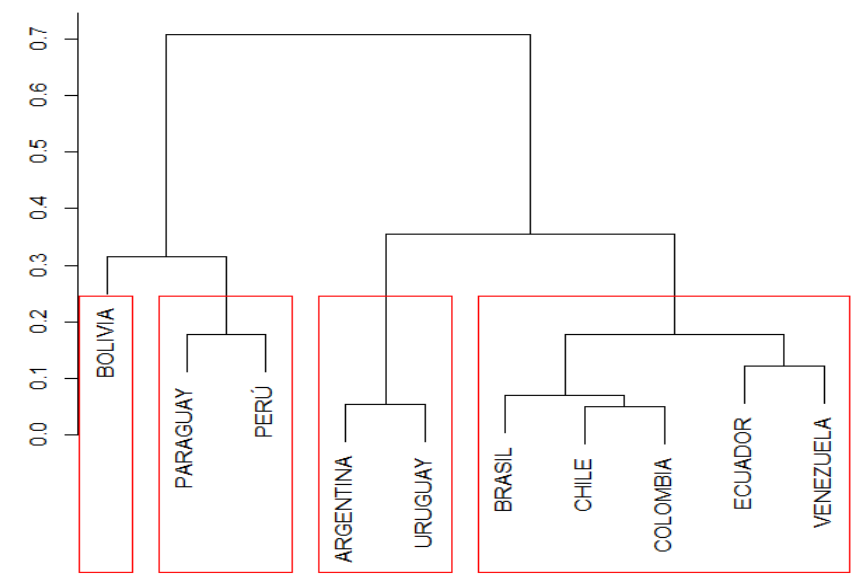

Fig. 2: Análisis Cluster de disponibilidad per cápita de leche en países de Sudamérica para décadas del 60 al 2000

\section{DISCUSIÓN}

El desarrollo de este estudio permitió evidenciar que la mayoría de las naciones de la región de Sudamérica presentaron diferencias estadísticas en la disponibilidad de leche a lo largo de las cinco décadas evaluadas (Tabla 1). En el informe anual sobre lechería FAO y FEPALE (2012) se reportó que la producción mundial de leche se ha incrementado, siendo que en 2011 se produjeron 614,4 millones de toneladas (Mt) que equivalen a un aumento del 2,5\% respecto al año anterior. Para el caso de Sudamérica, la producción se estimó en 
$68 \mathrm{Mt}$, representando un incremento del 5,5\% en la producción del alimento comparado con el año que lo antecede, donde la mayor producción se concentró en Brasil y Argentina que, además, fueron dos de los países que presentaron mayor cantidad de leche disponible (Tabla 1). A pesar del incremento de la producción lechera en los países de América del Sur, resultante del aumento del área dedicada a este fin y al número de vacas (Knipes, 2005), existe una brecha grande con la de Estados Unidos y la Unión Europea, regiones que presentan las mayores producciones.

Al analizar el consumo de leche, la literatura es consistente en afirmar que éste creció en las últimas décadas. Para los países en vía de desarrollo, dentro de los cuales se encuentran los sudamericanos, autores como Delgado (2003) reportan que en la década de los 80 y 90 el consumo per cápita de leche aumentó de 96 a 118g/persona-día (35 a 43kg/persona-año). En este mismo sentido, la FAO (FAO, 2008; FAO, 2010; FAO, 2012) reportó un incremento en la ingesta del alimento. Para 2007 el consumo fue 175g/persona-día (64kg/persona-año), en 2008 de 181g/persona-día (66 kg/persona-año) y para 2010 de 190g/persona-día $(69,4 \mathrm{~kg} /$ persona-año). Con base en lo anterior, se evidencia que el consumo per cápita de los países en vía de desarrollo presentó un incremento aproximado de 54,7\% entre la primera década del 2000 y la década del 90. Igual comportamiento fue encontrado por Sans y Combris (2015) en un estudio donde se evaluó el consumo de proteína de origen animal (ABP) en 183 países en el periodo entre 1961 y 2011, mostrando un aumento de 64g/persona-día en la década del 60 a $80 \mathrm{~g} /$ persona-día en la primera década del 2000 . Autores como Knipes (2005), Gerbens-Leenes et al. (2010), Rask y Rask (2011), Schneider et al. (2011) y, Sans y Combris (2015) reportaron que el incremento en la disponibilidad, producción e ingesta de alimentos en los países en vía de desarrollo se debe principalmente al crecimiento poblacional y al aumento de los ingresos per cápita (PIB). Este último factor a su vez, resulta en una mayor capacidad de compra de la población y en cambios sobre la cantidad y tipo de alimentos consumidos, es decir, cambios en los patrones de consumo. De esta forma, la población tiende a preferir el consumo de ABP como la leche, en vez de alimentos ricos en carbohidratos. De acuerdo con datos del World Bank (2019), las economías sudamericanas más desarrolladas (PIB $\geq$ US\$12000) son las de Chile, Argentina y Uruguay. Por el contrario, Bolivia (PIB < US\$ 3895) fue el país de la región que menos crecimiento económico tuvo. Lo anterior concuerda con los hallazgos de este estudio, en el cual se determinó que Uruguay y Argentina fueron los países que presentaron la mayor disponibilidad de leche mientras Bolivia la menor.

Aun cuando este estudio evidenció que $60 \%$ de los países sudamericanos exhibieron un incremento de la disponibilidad per cápita de leche a lo largo del tiempo evaluado (Tabla 2), dicha cantidad es insuficiente para satisfacer los requerimientos nutricionales de calcio. Según las recomendaciones de ingesta de nutrientes (García Gabarra et al., 2017; Ministerio de Salud y Protección Social, 2016; Ross et al., 2011) el consumo recomendado del mineral oscila entre los $800 \mathrm{mg}-1200 \mathrm{mg}$ para adultos, $1100 \mathrm{mg}-1300 \mathrm{mg}$ para adolescentes y 500mg-1000mg para niños menores de 10 años. Por tanto, para lograr aportes adecuados de calcio, se deben ingerir entre $600 \mathrm{~mL}-800 \mathrm{~mL}$ (3-4 porciones) que equivalen a $620 \mathrm{~g}-833 \mathrm{~g}$ de leche aproximadamente, lo cual es una cantidad mucho mayor a la disponible por país y por década, incluso en países como Argentina y Uruguay que presentaron la mayor disponibilidad per cápita (Tabla 1). Una revisión sistemática (Balk et al., 2018) encontró que las ingestas de calcio en las poblaciones del mundo son variables, oscilando entre 175 a $1233 \mathrm{mg} /$ día. Algunos países de Asia, África y América del Sur tienen un consumo del mineral por debajo de $500 \mathrm{mg}$, solo países del norte de Europa tienen una ingesta promedio mayor a 1000mg/día. Los datos anteriores reflejan que las poblaciones de los países desarrollados tienen un consumo de leche dentro de los valores de referencia y, además, presentan una ingesta de leche 3,49 veces mayor a la de los países en vía de desarrollo.

El consumo de productos lácteos presenta una gran variedad de beneficios para la salud humana. Según la revisión de Thorning et al. (2016), las personas con más baja ingesta de leche tienen mayor probabilidad de padecer obesidad. Los niños con ingestas más altas de leche y derivados tuvieron menos probabilidad (38\%) de sobrepeso y obesidad comparados con el grupo de menor consumo. El incremento de una porción de leche y derivados al día se asoció con menos masa grasa corporal y menos riesgo de sobrepeso y obesidad, lo cual podría ser explicado dado que estos productos son fuente de proteína que contribuye a la saciedad ayudando a prevenir una ingesta calórica mayor y, al ser una fuente importante de aminoácidos esenciales contribuye a la formación y mantenimiento del músculo que es considerado un tejido metabólicamente activo. De igual forma se evaluó la relación de la ingesta de leche y productos lácteos con el riesgo de diabetes tipo 2 y aunque no reportaron relación directa entre estos, si hacen referencia a un ligero beneficio para la salud previniendo la aparición de esta enfermedad. Existe una creciente evidencia que sugiere que los productos fermentados como yogurt y quesos están asociados con una reducción del riesgo de diabetes. Respecto al cáncer, la investigación ha demostrado que el consumo de leche y productos lácteos probablemente protege contra el cáncer colorrectal, de estómago y de seno, sin embargo, no determinó asociación con el riesgo de cáncer de próstata y ovario (Méndez et al., 2017). 
En relación a la salud ósea, está ampliamente documentado que el calcio, fósforo, magnesio, manganeso, zinc, vitaminas $\mathrm{D}$ y $\mathrm{K}$ son nutrientes necesarios para mantener un adecuado desarrollo del hueso dadas las interacciones positivas entre éstos (Ettinger, 2016) y, además, se encuentran en cantidades adecuadas en la leche y sus derivados. Es por lo anterior que una baja ingesta de estos nutrientes durante la infancia y adolescencia se asocia con un incremento significativo en el riesgo de osteoporosis y fracturas en la adultez temprana y mayor, especialmente en las mujeres (Van den Heuvel y Steijns, 2018).

Entre los años 2005 a 2012, la mayoría de países sudamericanos llevaron a cabo estudios sobre el consumo de alimentos y estado nutricional de la población, de forma tal que se pudieran establecer relaciones con enfermedades asociadas a la alimentación y nutrición, y a su vez, fueran la base para el diseño de políticas públicas. Con base en esas encuestas, se encontró que el consumo medio de leche y derivados lácteos de la población chilena fue de $335,7 \mathrm{~g} /$ persona-día $(325 \mathrm{~mL} /$ persona-día) resaltando que hay mayor consumo en niños que en adultos (Universidad de Chile, 2011). Argentina llevó a cabo su estudio en el 2007, no obstante, sólo reportó datos sobre cierta fracción de la población que incluyó niños, mujeres embarazadas y en edad fértil, impidiendo así, el reporte de datos sobre consumo a nivel poblacional (Ministerio de Salud, 2008). Por su parte, en Ecuador se reportó un consumo per cápita medio de derivados lácteos de 187g/persona-día (181 mL/persona-día) (Instituto Nacional de Estadísticas y Censos y Ministerio de Salud Pública, 2013).

En la encuesta sobre seguridad alimentaria y nutricional en municipios vulnerables realizada en Bolivia no se reportó un consumo real de leche y derivados, sin embargo, menciona que su consumo es muy bajo y que el $94 \%$ de los hogares no cubre ni el $90 \%$ de las recomendaciones mínimas de calcio (Programa Mundial de Alimentos-Ministerio de Desarrollo Agropecuario y Medio Ambiente, 2006). Venezuela presentó un consumo per cápita de derivados lácteos de 81,5g/persona-día (Instituto Nacional de Estadística, 2008) mientras que Perú exhibió un consumo anual de derivados lácteos líquidos de 19,7L/persona y de queso de 2,4kg/persona (Instituto Nacional de Estadísticas e Informática, 2009), representando en total un consumo per cápita de $62 \mathrm{~g} /$ persona-día. Colombia reportó un consumo per cápita de leche líquida y en polvo de $255 \mathrm{~g} /$ persona-día (Profamilia et al., 2006) y por su parte Brasil mostró que entre los años 2008-2009 su población tuvo un consumo de derivados lácteos cercano a 66g/persona-día (IBGE, 2011). Por último, de Uruguay y Paraguay no se encontraron encuestas reportadas. De acuerdo con la ingesta de leche y derivados reportados en las encuestas nacionales de ingesta de alimentos es posible observar que el consumo per cápita en la mayoría de países sudamericanos está por debajo de la media reportada por la FAO para los países en vía de desarrollo (FAO 2008; FAO 2010; FAO 2012) con excepción de Chile, Colombia y Ecuador. Es de resaltar que el consumo per cápita de lácteos en Brasil, Colombia, Ecuador, Perú y Venezuela está por debajo de la disponibilidad per cápita de leche (Tabla 1) lo que evidencia una doble problemática. Por un lado, la disponibilidad total de leche de los países analizados es insuficiente para alcanzar la recomendación nutricional y, por el otro, lo consumido por la población se encuentra por debajo de la cantidad disponible.

Las elecciones alimentarias están influenciadas por conocimientos, emociones, gustos, estilo de vida, experiencias anteriores con el alimento, entre otros, por ello, los patrones de consumo son moldeados por el entorno y la cultura del individuo (Leng et al., 2017). Un estudio realizado con adultos mayores en Suiza encontró que el consumo de leche y derivados podría estar limitado por preocupaciones y creencias frente al alimento. Se determinó que la mayoría de encuestados (60\%) se preocupan por el contenido de azúcares añadidos a estos productos, $28 \%$ por el contenido de grasa y $20 \%$ por la alergia a la proteína (Chollet et al., 2015). Otro estudio evidenció que una de las principales razones por los cuales los padres de niños y adolescentes no incluían productos lácteos en sus compras era por su elevado costo (Jung et al., 2017). Con base en lo anterior, se podrían establecer algunas estrategias que permitan fomentar la ingesta de leche y derivados lácteos en la población sudamericana. La educación nutricional ha demostrado ser una de las estrategias con mayor eficacia y por ello, investigadores del tema recomiendan educar a la población con información clara y objetiva, sustentada en la evidencia científica sobre las implicaciones para la salud debidas al bajo consumo de lácteos, desmitificar las creencias que se tienen alrededor del alimento y recomendar prácticas para lograr cambios de hábitos de forma exitosa (Jung et al., 2015).

De igual forma se recomienda un trabajo conjunto con la industria alimentaria con el fin de priorizar el diseño y la re-formulación de productos lácteos de alto valor nutricional adicionados con ingredientes más saludables. De otro lado, para mejorar la disponibilidad de leche se podrían plantear diferentes acciones que permitan incrementar la cantidad del alimento para consumo humano. De una parte, se podría sugerir mejorar la producción interna de leche a través de diferentes estrategias, tales como i) incrementar la cantidad de animales en producción (Knipes, 2005) lo que a su vez incrementaría el uso de recursos naturales (Rask y Rask, 2011; Schneider et al., 2011), ii) aumentar la productividad de los animales mediante mejoramiento genético (Knipes, 2005; Maitah y Smutka, 2012), iii) mejorar la tecnificación de los sistemas de producción de leche y procesamiento (Knipes, 2005; Maitah y Smutka, 2012), iv) intensificar la producción de leche (Knipes, 2005) y v) mejorar el precio de compra de leche a los productores (Knipes, 2005; Rich et al., 2011). Del otro lado, otra estrategia que se ha implementado en algunos países de Sudamérica como Colombia, es el 
establecimiento de tratados de libre comercio con regiones como Unión Europea o Estados Unidos que permitan incrementar la disponibilidad del alimento a través de la importación de mayores volúmenes de leche; no obstante, se debe considerar que dicha estrategia tiene un efecto negativo sobre la producción interna de leche ya que ésta no tiene cómo competir con el alimento proveniente de regiones con exportación subsidiada (Knipes, 2005; Maitah y Smutka, 2012).

\section{CONCLUSIONES}

Los hallazgos de este estudio permiten concluir que la disponibilidad de leche para consumo humano en los países de América del Sur aumentó a lo largo de los años. Sin embargo, existe una brecha entre lo disponible y la recomendación del alimento y, adicionalmente, la mayoría de los países presentó menor consumo respecto a la cantidad disponible. Las brechas existentes tanto en producción, disponibilidad e ingesta de leche en los países de la región sugieren el desarrollo de políticas públicas consistentes, que además de incentivar la producción del alimento, estén orientadas a fomentar su consumo, teniendo en cuenta su importancia para la salud humana.

\section{REFERENCIAS}

Abedini, M., E. Falahi y S. Roosta, Dairy Product Consumption and the Metabolic Syndrome, doi: 10.1016/j.dsx.2014.04.027, Diabetes Metab. Syndr., 9(1), 34-37 (2015)

Balk, E.M., G.P. Adam y otros ocho autores, Global Dietary Calcium Intake among Adults: a Systematic Review, doi: 10.1007/s00198-017-4230-x, Osteoporos. Int., 28(12), 3315-3324 (2018)

Chollet, M., D. Gille y otros cinco autores, Beliefs and Concerns about Dairy Products in the Swiss Older Adult Population, doi: 10.4172/2155-9600.1000365, J. Nutr. Food Sci., 5(3), 1-7 (2015)

Delgado, C.L., Rising Consumption of Meat and Milk in Developing Countries has created a New Food Revolution, doi: 10.1093/jn/133.11.3907S, J. Nutr., 133(11 Suppl 2), 3907S-3910S (2003)

Ettinger, S., Essentials III: Nutrients for Bone Structure and Calcification; in Nutritional Pathophysiology of Obesity and its Comorbidities. A Case-Study Approach by S. Ettinger, 271-284, Academic Press, New York City, USA (2016)

FAO y FEPALE, Federación Panamericana de Lechería, Situación de la Lechería en América Latina y el Caribe 2011: Informe Producido en el Ámbito del Observatorio de la Cadena Láctea de América Latina y el Caribe, FAO y FEPALE, Chile (2012)

FAO, Food and Agriculture Organization of the United Nations. Food Outlook Global Market Analysis. Noviembre de 2008 (en línea, acceso: 5 de mayo 2017), FAO, Italy (2008)

FAO, Food and Agriculture Organization of the United Nations, Food Outlook Global Market Analysis. Noviembre de 2010 (en línea, acceso: 5 de mayo 2017), FAO, Italy (2010)

FAO, Food and Agriculture Organization of the United Nations, Food Outlook Global Market Analysis. Noviembre de 2012 (en línea, acceso 5 de mayo 2017), FAO, Italy (2012)

FAO, Food and Agriculture Organization of the United Nations, Dietary Assessment a Resource Guide to Method Selection and Application in Low Resource Settings, 4-6, Rome, Italy (2018)

FAO, Food and Agriculture Organization of the United Nations, Food Outlook Global Market Analysis. Noviembre de 2018 (en línea, acceso 15 de enero 2019), FAO, Italy (2018)

FAOSTAT, Food Balance Sheet (en línea, acceso 15 de enero 2019), FAO, Italy (2019)

García Gabarra, A., M. Castellà Solley y A. Calleja Fernández, Ingestas de Nutrientes Recomendadas en la Unión Europea: 2008-2016, doi: 10.20960/nh.937, Nutr. Hosp., 34(2), 490-497 (2017)

Gerbens-Leenes, P.W., S. Nonhebel y M.S. Krol, Food Consumption Patterns and Economic Growth. Increasing Affluence and the Use of Natural Resources, doi:10.1016/j.appet.2010.09.013, Appetite, 55, 597-608 (2010)

IBGE, Instituto Brasileiro de Geografía e Estatística, Pesquisa de Orcamentos Familiares 2008-2009: Análise do Consumo Alimentar Pessoal no Brasil, IBGE, Brasil (2011)

Instituto Nacional de Estadística, Cuadro 5. Consumo Aparente Diario per Cápita, según Productos, Primer Semestre 2006-Segundo Semestre 2007, Instituto Nacional de Estadística, Venezuela (2008)

Instituto Nacional de Estadísticas e Informática, Consumo de Alimentos y Bebidas 2008, Instituto Nacional de Estadística e informática, Perú (2009)

Instituto Nacional de Estadísticas y Censos y Ministerio de Salud Pública, Encuesta Nacional de Salud y Nutrición ENSANUT 2012, Instituto Nacional de Estadísticas y Censos y Ministerio de Salud Pública, Ecuador (2013)

Jung, M.E., C. Mistry y otros cuatro autores, a Qualitative Investigation of Adults' Perceived Benefits, Barriers and Strategies for Consuming Milk and Milk Products, doi.org/10.1177/0017896914540295, Health Education Journal, 74(3), 364-378 (2015) 
Jung, M.E., J.E. Bourne, A. Buchholz y K.A. Martin Ginis, Strategies for Public Health Initiatives Targeting Dairy Consumption in Young Children: a Qualitative Formative Investigation of Parent Perceptions, doi: 10.1017/S1368980017002038, Public Health Nutrition, 20(16), 2893-2908 (2017)

Knipes, V., Developing Countries and the Global Dairy Sector Part I Global Overview, Food and Agriculture OrganizationAnimal Production and Health Division, Italia (2005)

Leng, G., R.A.H. Adan y otros once autores, the Determinants of Food Choice, doi: 10.1017/S002966511600286X, Proc. Nutr. Soc., 76(3), 316-327 (2017)

Lu, J., S. Zhang y otros cinco autores, Comparative Proteomics Analysis of Human and Ruminant Milk Serum Reveals Variation in Protection and Nutrition, doi: 10.1016/j.foodchem.2018.04.065, Food Chemistry, 261, 274-282 (2018)

Maitah, M. y L. Smutka, Economic Analysis of Milk Production and Consumption in the Middle East and North Africa, doi: 10.11118/actaun201260040245, Acta Univ. Agric. Silvic. Mendelianae Brun., 60, 245-254 (2012)

Méndez, V., G. Perdigón y A. de LeBlanc, Fermented Milks and Cancer in Dairy in Human Health and Disease Across the Lifespan by R.R. Watson; R.J. Collier y V.R. Preedy, 343-351, Academic Press, San Diego, USA (2017)

Ministerio de Salud y Protección Social, Resolución 3803 "Por la cual se establecen las Recomendaciones de Ingesta de Energía y Nutrientes-RIEN para la Población Colombiana y se Dictan Otras Disposiciones”, Ministerio de Salud y Protección Social, Colombia (2016)

Ministerio de Salud, Encuesta Nacional de Nutrición y Salud-ENNyS 2007, Ministerio de Salud, Argentina (2008)

Pereira, P.C. y F. Vicente, Milk Nutritive Role and Potential Benefits in Human Health; in Nutrients in Dairy and their Implications on Health and Disease by R.R. Watson; R.J. Collier y V.R. Preedy, 161-176 Academic Press, San Diego, USA (2017)

Pereira, P.C., Milk Nutritional Composition and its Role in Human Health, doi: 10.1016/j.nut.2013.10.011, Nutrition, 30(6), 619-627 (2014)

Profamilia, Instituto Nacional de Salud, Universidad de Antioquia e Instituto Colombiano de Bienestar Familiar, Encuesta Nacional de la Situación Nutricional en Colombia, 2005, $1^{\text {a }}$ Ed., 250-251, Panamericana formas e impresos S.A, Bogotá, Colombia (2006)

Programa Mundial de Alimentos-Ministerio de Desarrollo Agropecuario y Medio Ambiente, Resultados de la Encuesta de Seguridad Alimentaria y Nutricional en Municipios Vulnerables de Bolivia, Programa Mundial de Alimentos, Bolivia (2006)

Rask, K.J. y N. Rask, Economic Development and Food Production-Consumption Balance: A Growing Global Challenge, doi:10.1016/j.foodpol.2010.11.015, Food Policy, 36, 186-196 (2011)

Rich, K.M., R.B. Ross, A.D. Baker y A. Negassa, Quantifying Value Chain Analysis in the Context of Livestock Systems in Developing Countries, doi: 10.1016/j.foodpol.2010.11.018, Food Policy, 36, 214-222 (2011)

Ross, A.C., J.E. Manson y otros doce autores, The 2011 Report on Dietary Reference Intakes for Calcium and Vitamin D from the Institute of Medicine: What Clinicians need to Know, doi: 10.1210/jc.2010-2704, J. Clin. Endocrinol. Metab., 96(1), 53-58 (2011)

Rozenberg, S., J.J. Body y otros diez autores. Effects of Dairy Products Consumption on Health: Benefits and Beliefs-a Commentary from the Belgian Bone Club and the European Society for Clinical and Economic Aspects of Osteoporosis, Osteoarthritis and Musculoskeletal Diseases, doi: 10.1007/s00223-015-0062-x, Calcif. Tissue Int., 98(1), 1-17 (2016)

Sans, P. y P. Combris, World Meat Consumption Patterns: An Overview of the Last Fifty Years (1961-2011), doi: 10.1016/j.meatsci.2015.05.012, Meat Science, 109, 106-111 (2015)

Schneider, U.A., P. Havlík y otros nueve autores, Impacts of Population Growth, Economic Development, and Technical Change on Global Food Production and Consumption, doi: 10.1016/j.agsy.2010.11.003, Agricultural Systems, 104, 204215 (2011)

Secretaría de Economía-Dirección General de Industrias Básicas, Análisis del Sector Lácteo en México, Secretaría de Economía-Dirección General de Industrias Básicas, México (2012)

Simanca, M.M., R.D. Andrade y M.R. Arteaga, Efecto del Salvado de Trigo en las Propiedades Fisicoquímicas y Sensoriales del Yogurt de Leche de Búfala, doi: 10.4067/S0718-07642013000100010, Inf. Tecnol., 24(1), 79-86 (2013)

Thorning, T.K., A. Raben y otros cuatro autores, Milk and Dairy Products: Good or Bad for Human Health? an Assessment of the Totality of Scientific Evidence, doi: 10.3402/fnr.v60.32527, Food Nutr. Res., 60, 325-327 (2016)

Universidad de Chile, Encuesta Nacional de Consumo Alimentario-ENCA 2010, Universidad de Chile, Chile (2011)

Van den Heuvel, E.G.H.M. y J.M. Steijns, Dairy Products and Bone Health: How Strong is the Scientific Evidence?, doi: 10.1017/S095442241800001X, Nutr. Res. Rev., 21, 1-15 (2018)

World Bank, World Bank Country and Lending Groups, World Bank, USA (2019) 